\title{
Plasma Functionalization of Multiwalled Carbon Nanotubes and Their Use in the Preparation of Nylon 6-Based Nanohybrids
}

\author{
Roberto Scaffaro," Andrea Maio, Simonpietro Agnello, Antonella Glisenti
}

The possibility to obtain carbon nanotubes (CNT)/polyamide 6 composites with excellent mechanical properties in a simple, industrially scalable way is investigated. Commercial CNTs are treated by plasma while changing some key parameters (exposure time, plasma power, type of gas) in order to optimize the process and to achieve a sufficient degree of functionalization. The treated samples are characterized by Fourier transform infrared spectroscopy, Raman spectroscopy and X-ray photoelectron spectroscopy. The most interesting samples are selected to be used as reinforcing fillers, in different concentrations, in a polyamide 6 matrix. The mechanical tests show a dramatic increase of both tensile and impact properties, due to the achievement of a good wettability, and an efficient dispersion of the CNTs in the polymer matrix, as confirmed by scanning electron microscopy observations.

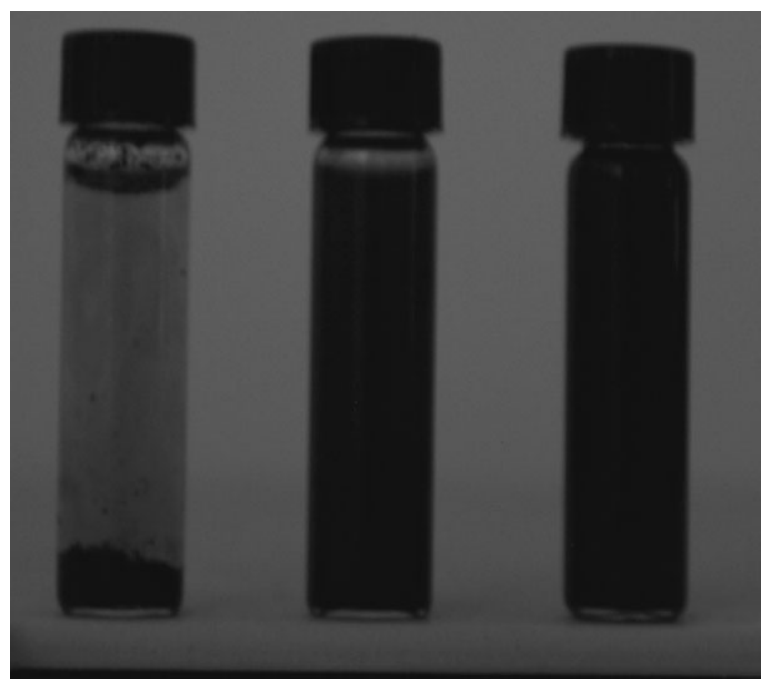

\section{Introduction}

Since their discovery, carbon nanotubes (CNTs) attracted remarkable interest, due to the extraordinary combination of relatively low density, large aspect ratio, good dimen-

R. Scaffaro, A. Maio

Dipartimento di Ingegneria Civile, Ambientale, Aerospaziale, University of Palermo, Viale delle Scienze, ed. 6, 90128, Palermo, Italy

E-mail: roberto.scaffaro@unipa.it

S. Agnello

Dipartimento di Fisica, University of Palermo, Via Archirafi, 36, 90123, Palermo, Italy

A. Glisenti

Dipartimento di Scienze Chimiche. University of Padova, Via

Marzolo, 1, 35131, Padova, Italy sional stability, high mechanical performance and electrical conductivity. All these features make this new class of nanoparticles attractive for their use as filler in polymer matrices to prepare multifunctional composites. ${ }^{[1,2]}$ One of the main problems that hinder a larger diffusion of CNTs as fillers is the commonly low affinity with most of polymer matrices.

Basically, the ways to incorporate CNTs into polymer matrices refer to two different inclusion approaches: in solution and in the melt. Methods bearing to the first category allow achieving the best results in terms of nanofiller dispersion but conversely they are expensive and may potentially cause problems in solvent removal/ recycle. Melt processing could represent the solution to reduce the costs and to speed up the manufacturing operations. Nevertheless, several problems, such as the prevention of CNTs bundling and the improvement of 
interfacial adhesion with polymer matrices, are still not completely solved.

Appropriate functionalization of CNTs may help to reduce their aggregation thus improving their dispersion within a polymer matrix. There are several approaches for the functionalization of CNTs, including defect functionalization, non-covalent functionalization, and covalent functionalization. ${ }^{[2-4]}$ Defect functionalization acts on the defective sites normally generated on the CNTs surfaces during their purification to introduce $-\mathrm{COOH}$ and $-\mathrm{OH}$ moieties. However, these sites are mainly located onto the tips surface and, therefore, their activity in promoting the dispersion and the unbundling in a polymer composite is generally poor. ${ }^{[3]}$

Non-covalent functionalization involves surfactants (anionic, cationic, non-ionic), biomacromolecules or wrapping with polymers to increase CNTs dispersion. ${ }^{[2]}$ In this case, the solubility and the processability are improved without compromising the physical properties of CNTs.

Covalent functionalization is based on the disruption of the translational symmetry of CNTs by converting $\mathrm{sp}^{2}$ carbon atoms to $\mathrm{sp}^{3}$. In particular, the $\pi$-orbitals of the $\mathrm{sp}^{2}$ hybridized $\mathrm{C}$ atoms, make CNTs quite reactive and prone to be grafted with other chemical species with potentially stronger adhesion CNTs/polymer and consequent improved mechanical performance. ${ }^{[4,5]}$ Typically, $-\mathrm{COOH}$ and $-\mathrm{OH}$ moieties are covalently induced following different pathways: oxidation by oxygen, air, aqueous hydrogen peroxide, concentrated sulfuric acid, nitric acid, or others acid mixtures. ${ }^{[6,7]}$ These treatments induce defects in the initially perfect lattice structure of the nanotube, thus allowing the formation of oxygenated groups onto the CNTs surface whose nature and amount depend on the type of treatment and on the operative parameters adopted. ${ }^{[8,9]}$ Furthermore, nanotube ends can be opened during the oxidation process. ${ }^{[10]}$

A more useful classification of functionalization methods can be adopted based on if a solvent is used (wet chemistry) or not (dry chemistry) during the modification process.

Wet chemistry methods traditionally consists of an oxidative step (using for instance concentrated acids) followed by eventual further steps in which the moieties are converted into other functionalities. Of course, wet chemistry approach exhibits the same problems described above about solution mixing: difficulty to remove the solvent, long reaction times requested and, in general, high costs, and high environmental impact.

Dry chemistry approaches include several techniques as crown discharge, photo-oxidation, radiation, and plasma treatments. Like all solvent-free systems, one of the main problem in adopting this method is the control of the homogeneity and of the degree of functionalization but these shortcomings are definitely counterbalanced by the rapidity of the process, as few minutes are often enough to achieve a good functionalization level.
Recent studies have been focused on the preparation of CNTs/thermoplastic polymers composites by melt mixing. Some authors prepared CNTs/PA6 composites by melt compounding, using functionalized CNTs ${ }^{[9,11]}$ Multiwalled carbon nanotubes (MWCNTs) were purified in $\mathrm{HCl}$ and then functionalized by refluxing them in $\mathrm{HNO}_{3}$. Mechanical tests showed that, compared with neat PA6, the elastic modulus improved more than $100 \%$ by incorporating 2 wt.\% CNTs. Chen et al. ${ }^{[12]}$ prepared reinforced PA6 by adding MWCNTs that were first oxidized, then acylated using $\mathrm{SOCl}_{2}$ and finally converted in amino-MWCNTs by reaction with hexamethylenediamine. The results remarked that, upon $0.5 \% \mathrm{w} / \mathrm{w}$, the inclusion of MWCNTs greatly improved mechanical and thermal properties of the composites. Uniform dispersion of MWCNTs and enhanced interfacial adhesion with the matrix were observed, likely due to the chemical reaction between carboxyl end groups of PA6 and $-\mathrm{NH}_{2}$ groups.

Xia et al. ${ }^{[13]}$ synthesized polymer-encapsulated MWCNTs through ultrasonically initiated in situ emulsion polymerization to use them as fillers for PA6. The Young's modulus was appreciably improved at $1 \mathrm{wt} . \%$ content of MWCNTs.

Aim of this work is the realization of high-performance nanohybrids materials based on polyamide 6 (PA6) and MWCNTs functionalized by plasma treatment. This method allows modifying the nanoparticles in few minutes without compromising the bulk structure. Moreover, this approach is solvent-free and it is easy to be controlled by tuning some key parameters (exposure time, plasma power, type of gas) in order to optimize the process and to achieve the desired functionalization level. The pathway that we propose for the realization of the nanohybrids is summarized in Figure 1.

FTIR, Raman and X-ray photoelectron spectroscopy (XPS) together with morphological analysis were carried out to demonstrate and quantify the CNTs functionalization. Blends of PA6 and functionalized MWCNTs were prepared in the melt and characterized from a morphological and mechanical point of view.

\section{Experimental Section}

\subsection{Materials}

In this study, an industrial grade of MWCNTs was supplied by Sigma-Aldrich ( $L=5-9 \mu \mathrm{m}, D=100-170 \mathrm{~nm}$, purity $>90 \%$ ). The polyamide 6 used for this work was a sample of Radilon S35 100 NAT, kindly supplied by Radicinova, Italy. It has an inherent viscosity (sulfuric acid) equal to $3.4 \mathrm{dl} \cdot \mathrm{g}^{-1}$. PA 6 was dried in a vacuum oven at $120^{\circ} \mathrm{C}$ overnight to prevent hydrolytic scission during processing.

\subsection{Functionalization of CNTs}

CNTs underwent plasma treatment in a Tucano Gambetti (Italy) radio-frequency plasma apparatus equipped with 2 mass flow 


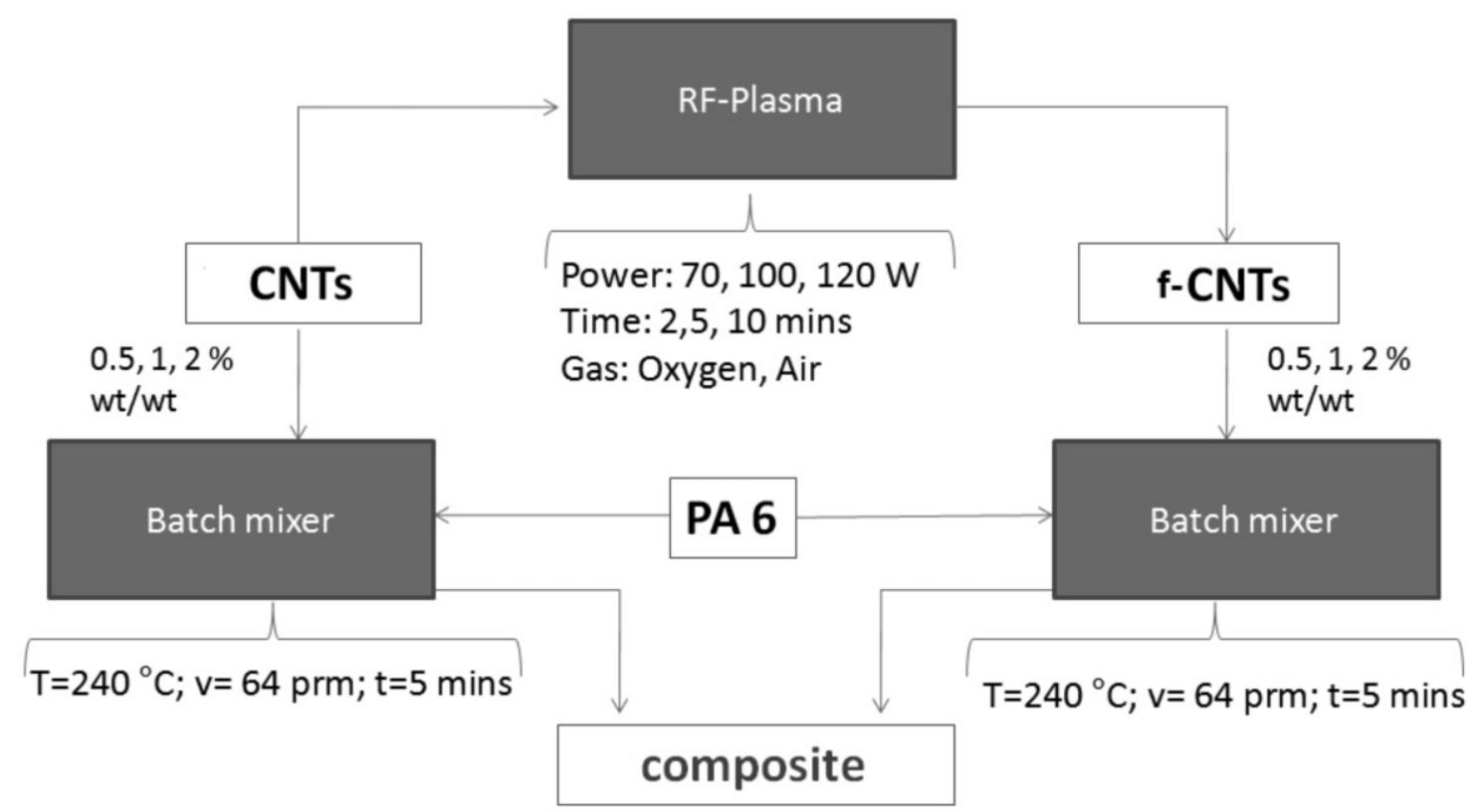

Figure 1. Schematic of the process and operative conditions adopted.

controllers, a dark-shield electrode and a stainless steel chamber. Several conditions, summarized in Figure 1, were used in order to get the optimum set of operative parameters. In this study, the exposure time was varied from 2 to $10 \mathrm{~min}$, plasma power from $70 \mathrm{~W}$ to $120 \mathrm{~W}$, the gases used were air and oxygen. In this latter case, the chamber was preventively prepared as follows: (i) vacuum was applied from room pressure to $0.2 \mathrm{mbar}$; (ii) oxygen was vented until 1 bar total pressure was achieved; (iii) vacuum was applied to achieve $0.2 \mathrm{mbar}$. In all the cases, the time of vacuum stabilization, i.e., at 0.2 mbar, was $5 \mathrm{~s}$. During the plasma treatment, after gas inlet (the pressure of the treatment gas was $0.3 \mathrm{mbar}$ ), the total pressure in the chamber was 0.5 mbar.

\subsection{Dispersion Tests}

The plasma treated CNTs (f-CNTs) were put in a flask together with distilled water $(0.5 \mathrm{~g} / \mathrm{L})$ and kept in an ultrasonic bath for $15 \mathrm{~min}$ at room temperature in order to impart an initial dispersion. The samples were then allowed to rest and the status of the dispersions was observed until $20 \mathrm{~d}$.

\subsection{Preparation of Composites}

PA6-CNTs and PA6-f-CNTs composites were obtained by melt mixing using an internal batch mixer. Neat (CNT), air-plasma (A-f-CNT) and oxygen-plasma (O-f-CNT) modified CNTs (0, 0.5, 1, and $2 \% \mathrm{w} / \mathrm{w}$ ) were added to PA6 in the solid state and then fed to a Brabender batch mixer until a constant value of the torque was achieved. The temperature was fixed at $240{ }^{\circ} \mathrm{C}$ and the rotational speed was set at $64 \mathrm{rpm}$. After processing, the material collected from the mixer was compression-molded in a Carver (USA) laboratory press adopting the following conditions: $P=100$ bar, $T=250^{\circ} \mathrm{C}$ for about $3 \mathrm{~min}$.

\subsection{Morphology and Energy Dispersive X-Ray Spectroscopy (EDS)}

The morphology of composites was observed by using a scanning electron microscopy (ESEM FEI OUANTA 200). The materials were fractured in liquid nitrogen and then sputtered with gold to ensure an electrically conductive surface.

TEM micrographs of CNTs and f-CNTs were obtained by using a high-resolution trasmission electron microscopy (HRTEM) JEM2100 (JEOL, Japan) at an accelerating voltage of $200 \mathrm{kV}$, equipped with an EDS analyzer. EDS was used to identify the elemental composition of both sidewalls and tips of the CNTs. The samples were dispersed in $2 \mathrm{ml}$ of 2-isopropanol, sonicated for $5 \mathrm{~min}$ and further deposed on a 300 mesh Holey carbon-coated nickel grids and allowed to dry overnight before examination.

\subsection{Fourier Transform Infrared (FT-IR), Raman and X-Ray Photoelectron Spectroscopy (XPS)}

FT-IR analysis was performed by using a Perkin-Elmer FT-IR/NIR Spectrum 400 spectrophotometer, the spectra were recorded in the range $4000-400 \mathrm{~cm}^{-1}$.

Micro-Raman spectroscopy was carried out at room temperature by a Bruker-Senterra micro-Raman equipped with a $532 \mathrm{~nm}$ diode laser excitation with $20 \mathrm{~mW}$ power. Non-confocal measurements were carried out in the range $4000-1000 \mathrm{~cm}^{-1}$ with a spectral resolution between 9 and $15 \mathrm{~cm}^{-1}$.

The XPS investigations were carried out with a Perkin-Elmer $\Phi$ 5600ci Multi Technique System. The spectrometer was calibrated 
by assuming the binding energy $(\mathrm{BE})$ of the $\mathrm{Au} 4 \mathrm{f}_{7 / 2}$ line to be $84.0 \mathrm{eV}$ with respect to the Fermi level. Both extended spectra (survey $-187.85 \mathrm{eV}$ pass energy, $0.5 \mathrm{eV} \cdot$ step $^{-1}, 0.05 \mathrm{~s} \cdot \mathrm{step}^{-1}$ ) and detailed spectra (for $\mathrm{C} 1 \mathrm{~s}$ and $\mathrm{O} 1 \mathrm{~s}-11.75 \mathrm{eV}$ pass energy, $0.05 \mathrm{eV} \cdot$ step $^{-1}, 0.2 \mathrm{~s} \cdot$ step $^{-1}$ ) were collected with a standard $\mathrm{Al}$ $\mathrm{K} \alpha$ source working at $300 \mathrm{~W}$. The standard deviation in the $\mathrm{BE}$ values of the XPS line is $0.10 \mathrm{eV}$. The atomic percentage, after a Shirley-type background subtraction, ${ }^{[14]}$ was evaluated by using the PHI sensitivity factors. ${ }^{[15]}$ The peak positions were corrected for eventual charging effects by considering the $C 1$ s peak at $284.8 \mathrm{eV}$ and evaluating the BE differences. ${ }^{[16]}$ The curve fitting was carried out by means of an iterative least square procedure making use of Voight functions on Shirley-type background. ${ }^{[16]}$

\subsection{Mechanical Characterization}

The sheets realized were cut to obtain specimens for mechanical characterization: according to ASTM D882 for tensile tests and according to ASTM D256 for impact tests. Tensile tests were carried out on an Instron 3365 (USA) universal testing machine with a crosshead speed of $5 \mathrm{~mm} / \mathrm{min}$ and $30 \mathrm{~mm}$ of initial gauge length on at least ten replicates. Impact tests were carried out by a Ceast mod. 6545 (Italy) universal apparatus on at least seven replicates of notched samples in the Izod mode. All the data were processed by calculating the average values and reporting the related error bars taking into account the data scattering.

\section{Results and Discussion}

The dispersion stability of nanotubes is an important factor in fabricating uniformly dispersed CNTs composites.

Figure 2 shows air-functionalized (A-f-CNTs), oxygenfunctionalized (O-f-CNTs) and as received CNTs dispersed in water after sonication, according to the methodology described in the experimental part. The picture puts into evidence that raw CNTs have poor dispersion stability in water, as they almost completely settled just after $<1 \mathrm{~h}$ after sonication. Differently, f-CNTs, remain in aqueous suspension, regardless of the type of gas used for the plasma treatment. As already found in other works, this is likely due to the formation of hydrophilic groups (as $-\mathrm{COOH},-\mathrm{OH}$ ), which, in their dissociated form, stabilize the nanotubes by an electrostatic stabilization mechanism and prevent or limit the formation of bundles. ${ }^{[17]}$

In Figure 3, there are reported the TEM micrographs obtained for all the samples at different magnifications. Neat CNTs (Figure 3a) are evidentially organized in bundles with single CNTs having a 200-400 nm diameter and a length of about 1-10 $\mu \mathrm{m}$. A-f-CNTs, Figure 3b, and O-f-CNTs, Figure $3 c$, have substantially the same dimensions, the surface morphology is similar, thus confirming that plasma treatment does not cause drastic changes in the CNTs structure. Anyway, in this case it is worth noting that only few aggregates have been observed for A-f-CNTs, as in Figure $3 \mathrm{~b}$, and it was possible to find a large number of

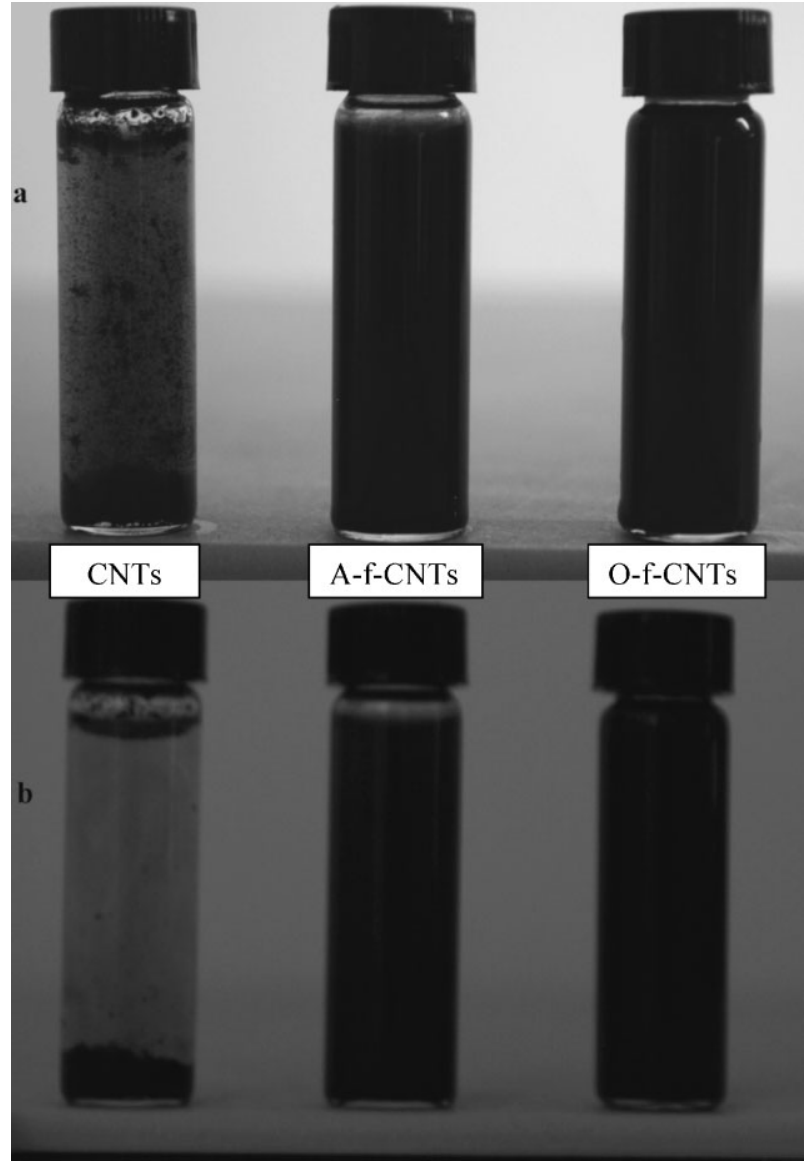

Figure 2. Dispersion stability in water of CNTs, A-f-CNTs, O-f-CNTs after $2 \mathrm{~h}(\mathrm{a})$ and after 20 days (b).

isolated nanotubes for O-f-CNTs sample, as in Figure 3c. Again, the presence of hydrophilic groups (as $-\mathrm{COOH},-\mathrm{OH}$ ) is supposed to stabilize the colloidal suspensions in 2-isopropanol by electrostatic mechanism, as discussed in the case of water dispersion. ${ }^{[17-19]}$

EDS analysis put into evidence the presence of $\mathrm{Ni}, \mathrm{C}$, and $\mathrm{Cu}$ (due to the grid used to hold the samples during TEM observation), Fe (attributable to the catalyst used for the CNTs synthesis) and $\mathrm{O}$ (attributable to alcoholic and carboxylic moieties for the plasma treated samples both in tips and sidewalls). Actually, small amounts of oxygen are present also in the pristine CNTs. This can be explained with the presence of defect sites in the transition zone between the fullerenic tips and the graphenic walls. ${ }^{[20]}$

Figure 4 reports the FT-IR spectra of pristine CNTs (a) A-fCNTs (b) and O-f-CNTs (c). Although the baseline is bad and some parts of the spectrum are not well defined, it is possible to individuate some differences between the two $\mathrm{f}$-CNT samples. In particular, in the range $1800-1000 \mathrm{~cm}^{-1}$, it is possible to observe a broad signal both for A-f-CNTs and O-f-CNTs, not present in the spectrum of neat CNTs and attributable to carboxylic moieties (in the anionic 


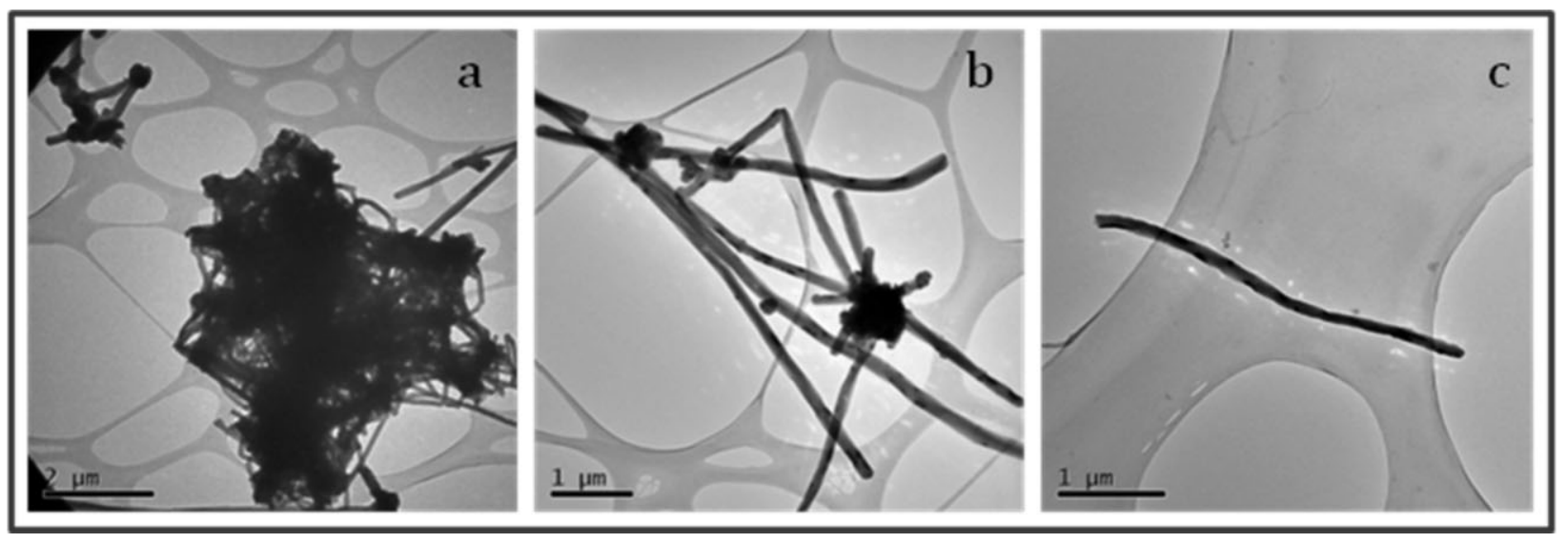

Figure 3. TEM micrographs of the (a) CNTs, (b) A-f-CNTs and (c) O-f-CNTs dispersions in 2-isopropanol.

form). ${ }^{[19,20]}$ More in detail, the two bands at around $1600 \mathrm{~cm}^{-1}(\mathrm{C}=\mathrm{O})$ and $1100 \mathrm{~cm}^{-1}(\mathrm{C}-\mathrm{O})$, correspond to the stretching mode of the carboxylic acid group $(-\mathrm{COOH})$.

This analysis confirms that plasma treatment induced some changes on the CNTs surface even if it is not sufficient to get conclusive and quantitative data. The investigation about the influence of the different treatment parameters on the functionalization is a complex issue that requires, therefore, a multi-technique analysis approach. For this reason, Raman spectroscopy and XPS analysis were carried out too.

In Figure 5, it is reported the Raman spectra of CNTs, A-f-CNTs, and O-f-CNTs, normalized to the amplitude of the G-band. It is possible to see evident bands at 1339 , 1569, 2680 and $3220 \mathrm{~cm}^{-1}$, in good agreement with the structures typically associated, respectively, to D-band, G-band, $G^{\prime}$-band, and 2G-band. ${ }^{[21,22]}$ It is worth noting that the spectra, at wavenumbers higher than $2300 \mathrm{~cm}^{-1}$, are

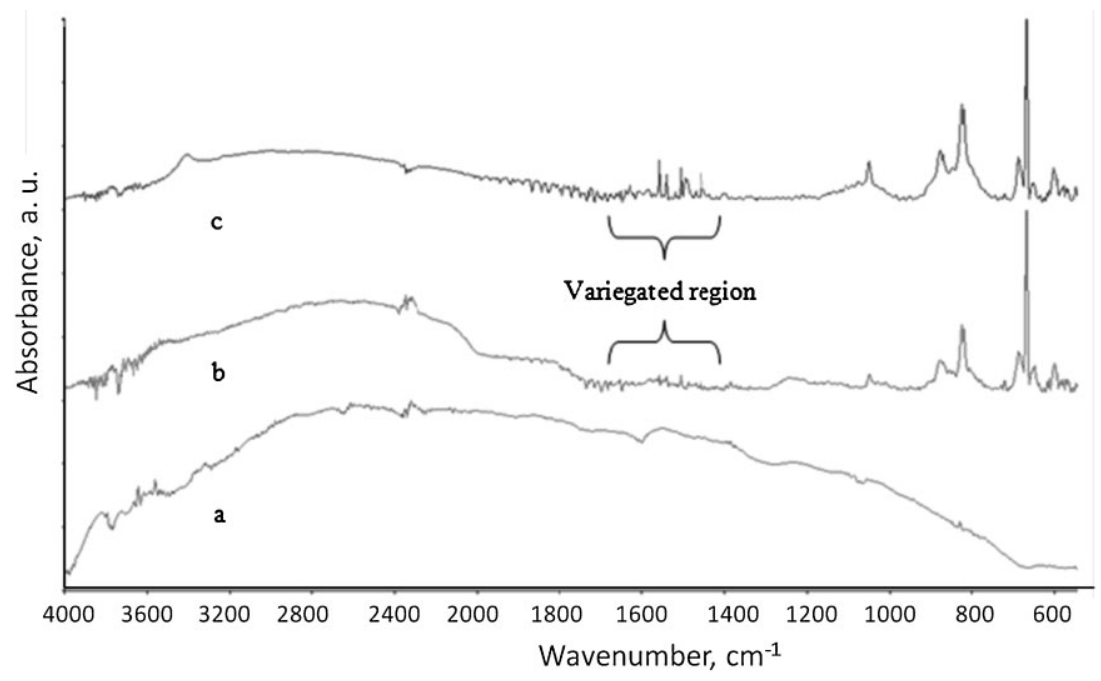

Figure 4. FT-IR spectra (adsorbance) of CNTs (a), A-f-CNTs (b) and O-f-CNTs (c). practically coincident independently on the different treatments undergone by the samples. In other words, the normalization of the different spectra can be performed using the $G$, the $G^{\prime}$ or the $2 G$ band without any difference. This, of course, makes the comparison between the measurements more than reliable.

Particular attention must be paid to the changes of the ratio of the amplitudes of the $G$ and the $D$ bands (IG/ID). This ratio can be correlated with the degree of functionalization by the different vibration mode of carbon atoms in the presence of defects, generated during the functionalization process and associated to the presence of oxygen or other atoms in the nanotube structure. ${ }^{[21-23]}$ In particular, the G-band is associated to the stretching of $\mathrm{C}-\mathrm{C}$ bonds in graphitic materials ( $\mathrm{sp}^{2}$ hybridization) while the $\mathrm{D}$-band is associated with phonons close to the boundary of the Brillouin zone, usually detectable by Raman for disordered crystals (defects in $\mathrm{sp}^{2}$ carbons). By comparing the spectra of functionalized and unfunctionalized CNT, Figure 5, it is well evident that the ID/IG ratio sensibly increases for the formers with respect to that observed for neat CNTs. In particular, ID/IG for O-fCNTs sample is dramatically higher than that observed for neat CNTs. In Figure 6, it is reported the ID/IG ratio as a function of power for sample treated under different gas media. The ID/IG ratio monotonically increases for O-f-CNTs, while it presents a maximum at $100 \mathrm{~W}$ for A-f-CNTs.

This apparently strange behavior can be explained considering the mechanism of modification of CNTs during plasma functionalization. As reported by other authors, ${ }^{[22,23]}$ and summarized in the scheme reported in Figure 7 , during the plasma treatment two phenomena 


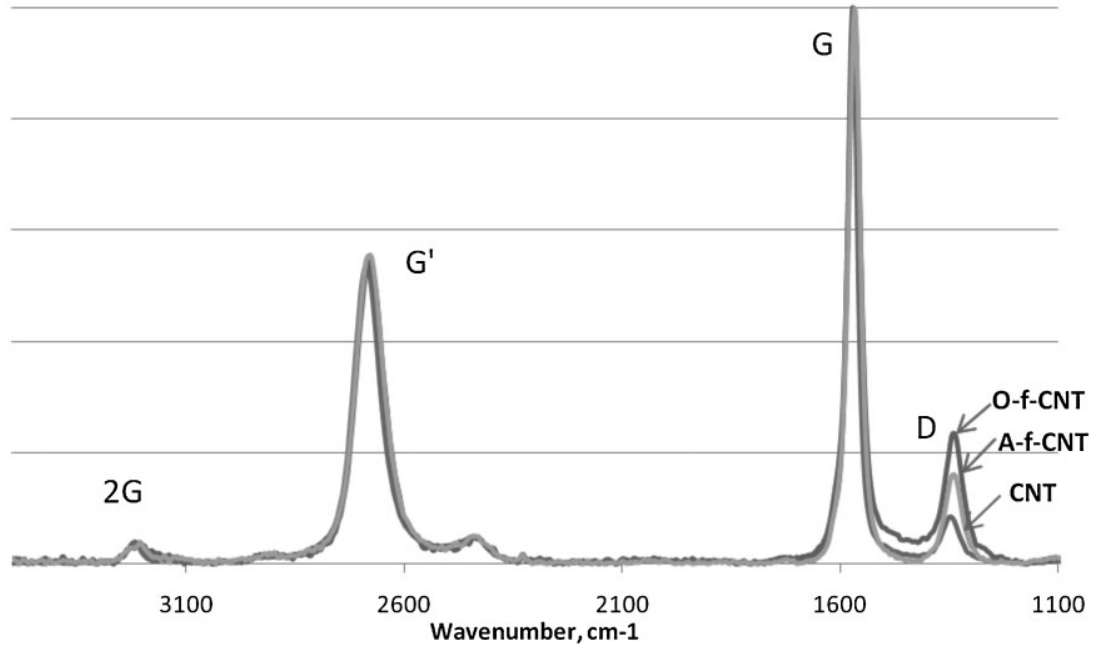

Figure 5. Raman spectra of CNTs, A-f-CNTs and O-f-CNTs (plasma treated at $120 \mathrm{~W}$ for $10 \mathrm{~min})$.

occur: (i) oxygen groups formation, (ii) oxygen functionality loss by formation of volatile oxygen-containing products (walls ablation). These two phenomena are always concurrent during the treatment and they have two opposite effects on the ID/IG ratio. In particular, if the conversion of $\mathrm{sp}^{2}$ into $\mathrm{sp}^{3}$ carbons prevails - due to the oxygenated groups formation onto the CNTs surface - an increase of the ID/IG ratio is observed. On the other hand, the gasification of the amorphous carbon and, generally, of the nanotube walls, causes a decrease of ID/IG. ${ }^{[22,23]}$ In other words, a lower or higher value of this parameter does not indicate an absolute modification level of the CNTs (i.e., higher defects or higher ablation) but only that the phenomenon " $\mathrm{i}$ " was relatively less or more intense than the phenomenon "ii".

In our case, for A-f-CNTs samples, it can be supposed that, below $100 \mathrm{~W}$, the kinetic of oxygenated groups growth (correlated to the defects) is faster than that of surface ablation. Differently, when oxygen is used, the correspond-

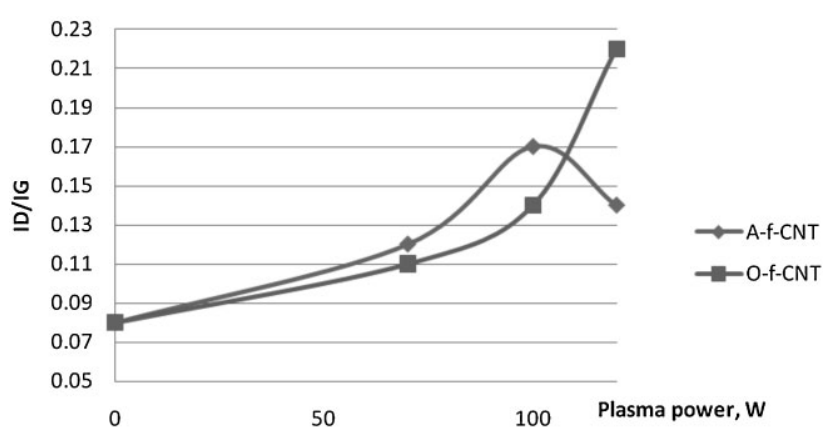

Figure 6. Raman ID/IG. Effect of power and gas. ing O-f-CNTs, show a monotonic increase of ID/IG in the whole power range here investigated. In this case, it may be hypothesized that the kinetic of formation of defects, on which $-\mathrm{OH},-\mathrm{COOH}$ and $\mathrm{C}=\mathrm{O}$ are formed, is supposed to be faster than the ablation of the outer walls of the CNTs.

Although Raman spectroscopy provides more detailed and precise information compared with FTIR, it would be useful to have further confirmation and verification of the results obtained by using a direct and quantitative analytical method.

Plasma functionalization is a surface phenomenon; because of this reason XPS was also used.

Figure 8 reports the XPS survey spectra of pristine CNTs, A-f-CNTs and O-f-CNTs (both treated at $120 \mathrm{~W}$ and $t=600 \mathrm{~s}$ ). The spectra only reveal the presence of carbon and oxygen; in particular no traces of nitrogen are observed.

In Table 1, the XPS atomic compositions are reported. Oxygen is scarcely present in pristine CNTs. The small amounts measured are likely due to the preparation and manipulation of the materials prior to their commercialization, ${ }^{[1-13,17-26]}$ and according to the EDS analysis, the amount of oxygen is, as expected, definitely higher in the two functionalized samples, especially in O-f-CNTs.

In Figure 9a, the high-resolution spectra of $O 1$ s for neat CNTs and for O-f-CNTs, and A-f-CNTs samples, are shown. The comparison among the spectra of the two functiona-
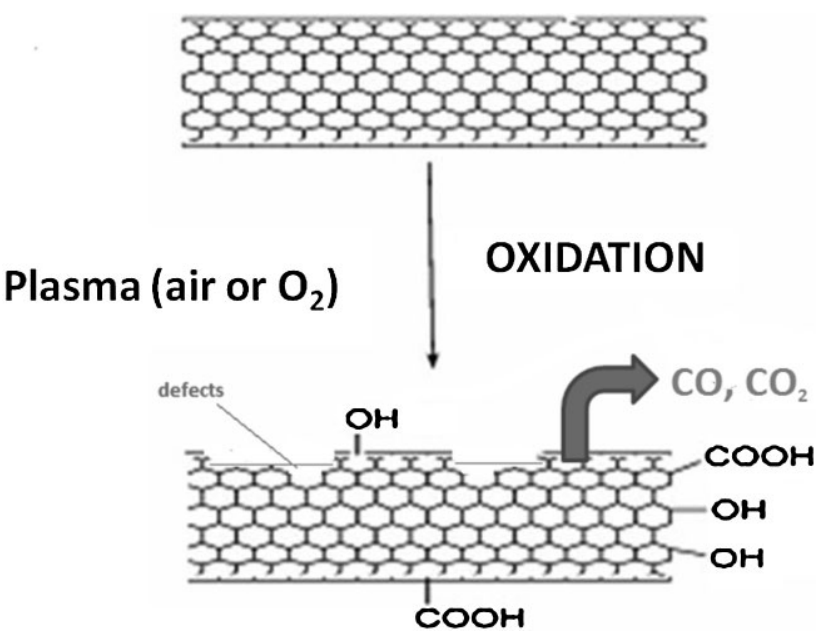

Figure 7. Possible mechanism of modification by a plasma: grafting of oxygenated moieties and ablation of carbonaceous products (converted to $\mathrm{CO}_{2}$ ). 


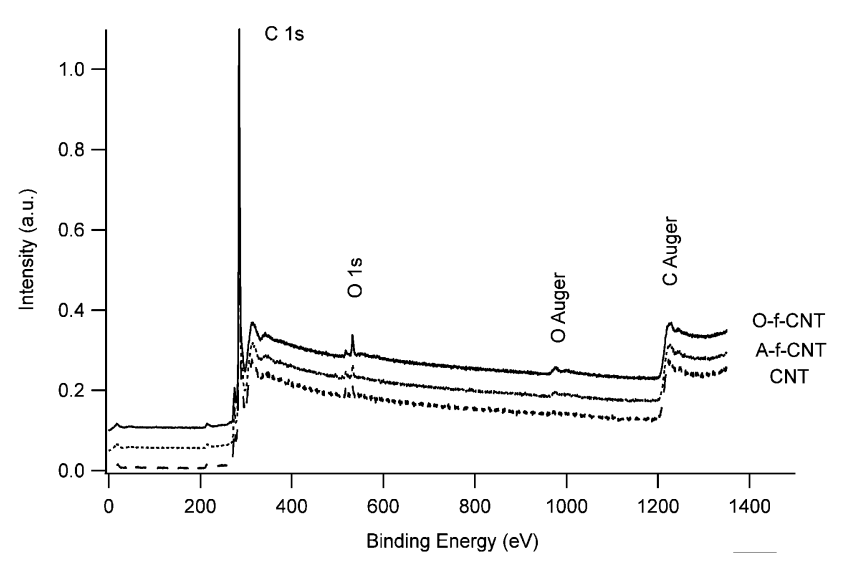

Figure 8. Survey XP spectra of CNTs (dashed line), A-f-CNTs (dotted line) and O-f-CNTs (continuous line).

Table 1. Elemental composition of the CNTs samples object of this study as obtained by XPS.

\begin{tabular}{lll}
\hline Sample & C [\%] & O [\%] \\
\hline CNT & 98.7 & 1.3 \\
A-f-CNT & 94 & 6 \\
O-f-CNT & 90.6 & 9.4
\end{tabular}

lized samples reveals a slight shift toward higher BE for the peak of the O-f-CNTs sample; this behavior suggest a more significant presence of $\mathrm{C}-\mathrm{O}-\mathrm{C}$ groups and/or carboxylic groups (533.0-533.5 eV) whereas a more evident contribution of $\mathrm{C}=\mathrm{O}$ species $(532.2-532.8 \mathrm{eV})$ seems in agreement with the A-f-CNTs peak.

In Figure 9b, the high-resolution C 1s spectra are reported. The fitting procedure reveals the presence of different contributions and, in particular, $\mathrm{C}-\mathrm{C}, \mathrm{C}=\mathrm{C}$ and $\mathrm{C}-\mathrm{H}$ $(284.8 \mathrm{eV}) ; \mathrm{C}-\mathrm{O}(286.0-286.3 \mathrm{eV}), \mathrm{O}-\mathrm{C}=\mathrm{O}^{*}(289-290 \mathrm{eV})$ $\pi-\pi^{*}$ shake-up satellite peaks $(292 \mathrm{eV})$ ascribed to intact phenyl rings of the CNT structure. The accurate comparison among the C1s signals obtained for the different samples, reveals a more evident presence of carbon species bound to oxygen. This observation is also confirmed by fitting data (Table 2).

It can also be observed that, despite relative magnitude, an increase of total oxygen (measured by XPS) corresponds to an increase of the ID/IG ratio (obtained by Raman). This important result allowed using this latter as a qualitative measure of functionalization level.

A-f-CNTs and O-f-CNTs treated for 10 min at 70 and $120 \mathrm{~W}$ were selected for the preparation of PA6/CNTs composites.

In Figure 10a-c, there are reported the tensile mechanical properties as a function of CNT content for the different f-CNTs used in the frame of this study, together with neat CNTs for comparison.
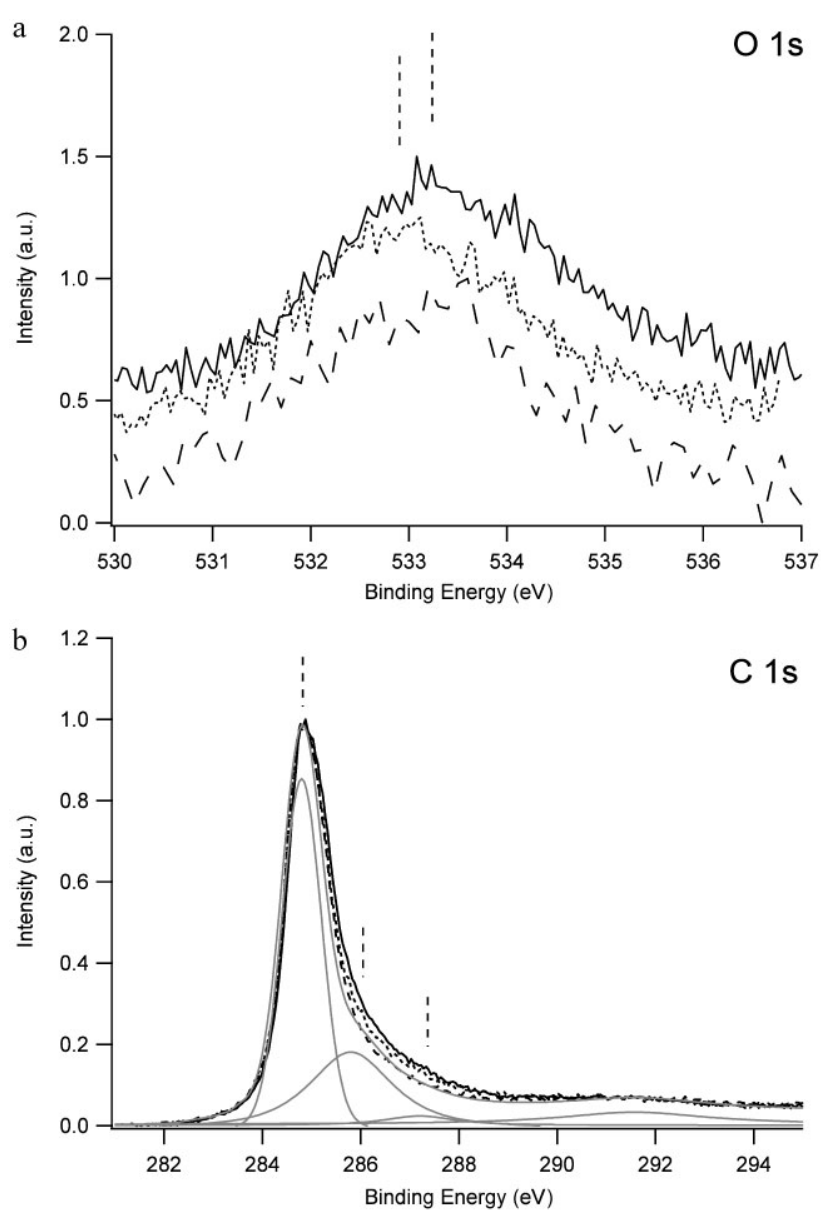

Figure 9. O1s (a) and C1s (b) XP spectra of CNTs (dashed line), A-f-CNts (dotted line), and O-f-CNTs (continuous line). The fitting results obtained for CNTs is also reported as an example.

Table 2. Normalized values of $\mathrm{C}$ is atomic concentrations of the different components.

\begin{tabular}{llrcc}
\multirow{2}{*}{ Components } & $\begin{array}{c}\text { Position } \\
{[\mathrm{eV}]}\end{array}$ & \multicolumn{3}{c}{ Atomic concentration [\%] } \\
\cline { 3 - 5 } & & CNT & A-f-CNT & O-f-CNT \\
\hline $\mathrm{C}-\mathrm{C}, \mathrm{C}=\mathrm{C}, \mathrm{C}-\mathrm{H}$ & 285.4 & 49 & 52 & 37 \\
$\mathrm{C}-\mathrm{O}$ & 286 & 45 & 43 & 54 \\
$\mathrm{O}-\mathrm{C}=\mathrm{O}$ & 287.8 & 2 & - & 6 \\
$\pi-\pi^{*}$ & 291.8 & 4 & 5 & 3
\end{tabular}

For all the CNTs used, the most relevant increment of the mechanical properties is observed when only $0.5 \%$ by weight of CNTs is added. Above this CNTs level, the increase is modest and tends to level off after $1 \% \mathrm{w} / \mathrm{w}$ for all the CNTs used. Adding a small amount of CNTs causes a remarkable increase in elastic modulus in the composites, due to the hindered mobility of the polymer chains and to the intrinsic 

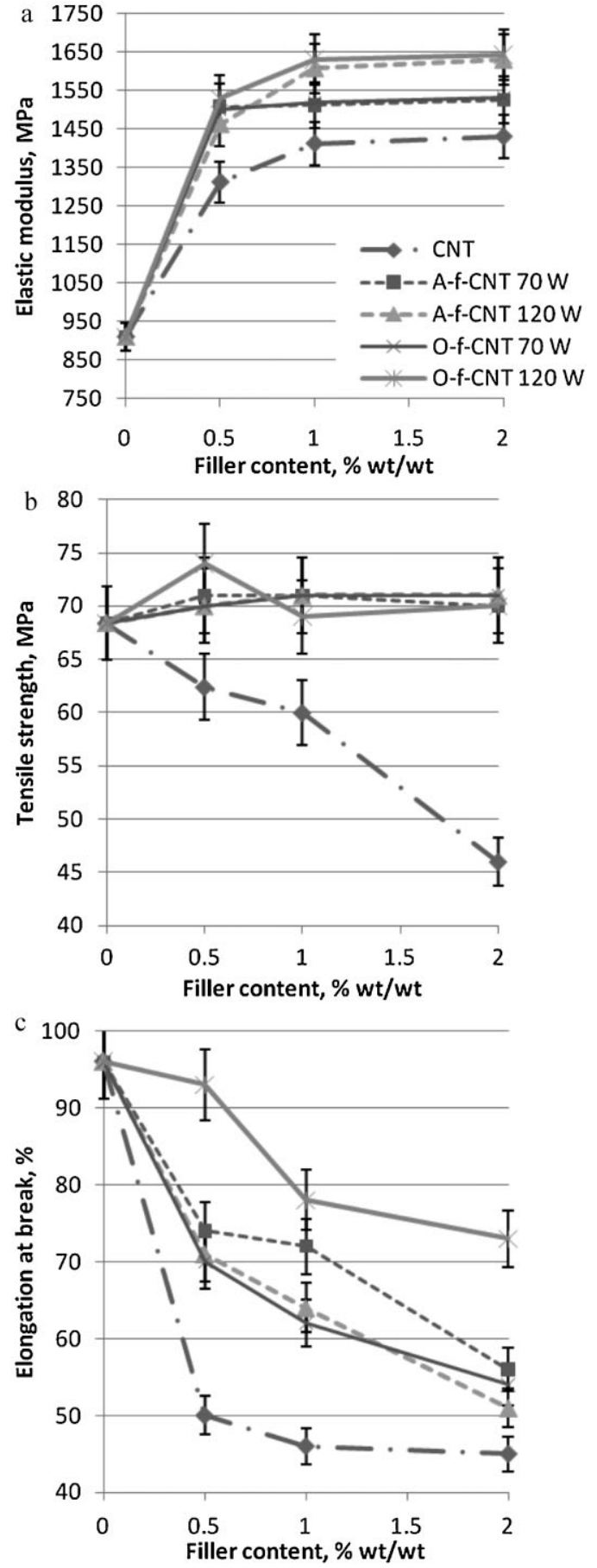

Figure 10. Elastic modulus (a), tensile strength (b) and Elongation at break (c) for various type of PA6/CNTs composites as function of filler content ( $\mathrm{f}$-CNTs modified under different plasma conditions).

reinforcing effect of the CNTs. The situation is even better when f-CNTs, and in particular O-f-CNTs, are used. In this case, the elastic modulus is about $85 \%$ higher than that of the neat matrix and about 30\% higher than that of PA6/
CNTs. Composites containing A-f-CNTs show values substantially similar to O-f-CNTs even if slightly lower.

Some difference must be noted for the effect of the power as the composites containing the nanotubes functionalized at the highest power $(120 \mathrm{~W})$ exhibit the highest values of this property. This feature can be explained considering that the presence of hydrophilic groups onto the surface of functionalized nanotubes, promotes the adhesion between the filler and the matrix with better stress transmission between the two phases, with positive consequences on the mechanical performance.

These results are corroborated by the analysis of the tensile stress, Figure $10 \mathrm{~b}$ and the elongation at break, Figure 10c.

In a composite material, the increase of rigidity due to the presence of a filler, some bad interfacial adhesion between the filler and the matrix and the presence of inclusion caused by a bad dispersion are usually responsible of a decrease of the breaking properties.

Adding CNTs caused a decrease of the tensile stress, Figure 10b. Probably the high-melt viscosity of the composite hindered the dispersion of the CNTs in the matrix, as found by Chen et al. ${ }^{[12]}$ for amino-MWCNTs/PA6 composites in which they measured even a decrease both in elastic modulus and tensile strength.

When f-CNTs are added, the tensile stress remains practically constant at any concentration. These data can be interpreted considering that the oxygenated moieties on $\mathrm{f}$-CNTs, induced a better adhesion with the matrix and, consequently, higher ultimate resistance is achieved if compared with that of composites containing unfunctionalized CNTs. Elongation at break, Figure 10c, decreases on increasing nanofiller concentration both for CNTs and for f-CNTs. It is worth observing, however, that the worsening of this property is less dramatic when $\mathrm{f}$-CNTs are used. Again, O-f-CNTs prepared at $120 \mathrm{~W}$ showed the best performance.

In order to have a confirmation about improvement of the dispersion and of the adhesion in f-CNTs based composites, SEM images of the cross section of materials fractured in liquid nitrogen have been obtained and reported in Figure 11.

The morphology of the fracture surface of the samples puts into evidence that the materials containing unfunctionalized CNTs, Figure 11a, present lack of adhesion matrix/filler and the evident presence of CNTs bundles. On the contrary, it is well evident the good wetting of A-fCNTs, Figure 11b, and O-f-CNTs, Figure 11c, that are well embedded in the matrix, in full agreement with the mechanical data presented above.

The CNTs used in this work have a diameter of approximately $200 \mathrm{~nm}$ and, therefore, the dispersion is theoretically granted and the presence of hydrophilic moieties on the sidewalls of CNTs is likely to further 

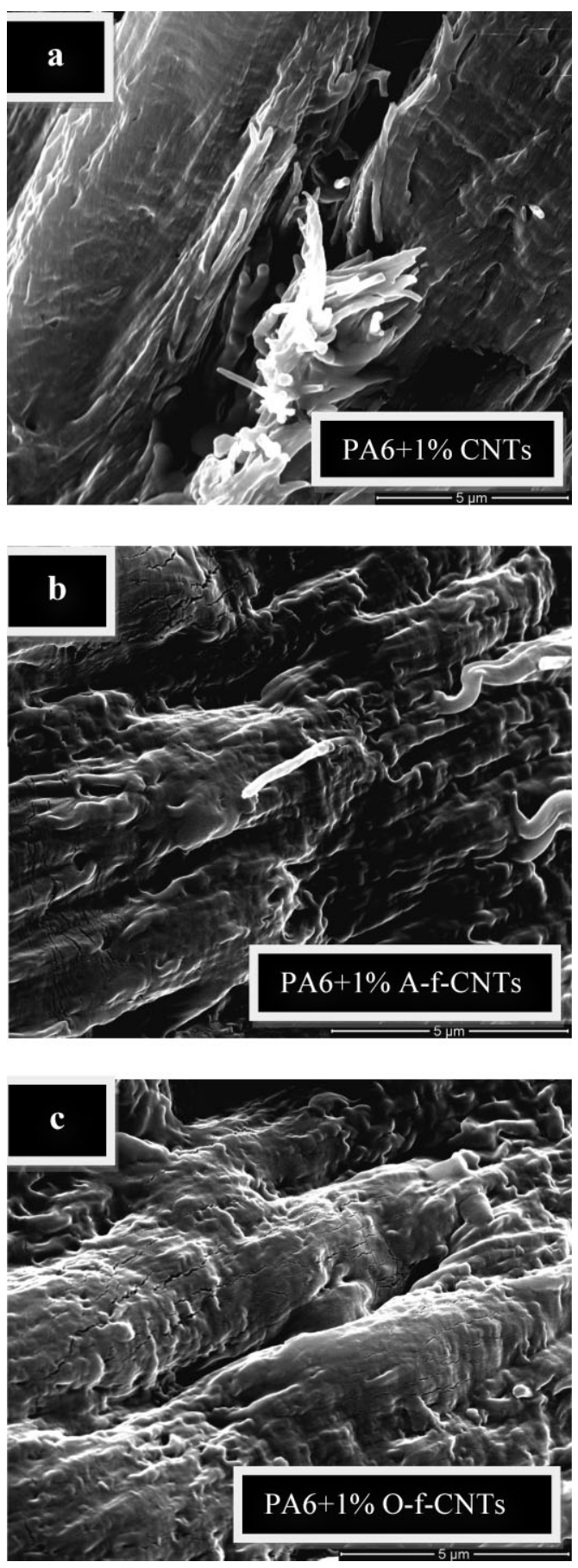

Figure 11. SEM micrographs of composites containing $1 \%$ of (a) CNTs, (b) A-f-CNTs, (c) O-f-CNTs.

increase their affinity to PA6. Moreover, the functionalization could cause a decrease of the bulk density of CNTs so to improve the ability of these materials to be dispersed. ${ }^{[2-30]}$

Figure 12 reports the impact strength recorded for PA6 filled materials with a content of $1 \% \mathrm{w} / \mathrm{w}$ CNTs or f-CNTs together with the values of pure PA6, for comparison. In

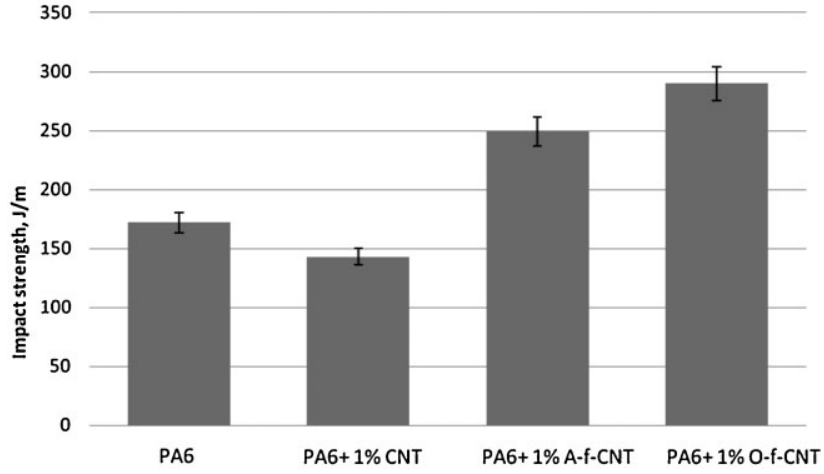

Figure 12. Izod tests for PA6 and composites containing $1 \%$ of CNTs, A-f-CNTs, O-f-CNTs (f-CNTs treated at $120 \mathrm{~W}$ for $10 \mathrm{~min}$ ).

general, f-CNT based materials display a better resilience than those containing neat CNTs. The inclusion of CNTs in the polymer matrix, as depicted in Figure 12, can be considered the responsible of the worsening of the impact properties even at low concentrations. The situation is different when A-f-CNTs and especially O-f-CNTs are used. In this case, the values of impact strength are about five times higher than those measured for pure PA6, indicating a strong interaction between the matrix and the filler. The considerations discussed before are valid also to interpret the impact tests results. Again, the reasons of this behavior can be again attributed to a better wettability and, in general, to a better interaction between matrix and nanofiller which led to a stronger interfacial adhesion.

\section{Conclusion}

In this work, MWCNTs were functionalized adopting a dry chemistry methodology. In particular, air and oxygen plasma were used to introduce oxygenated moieties onto the MWCNTs surface.

FTIR, Raman and XPS spectroscopical analysis revealed that oxygen plasma is more effective than air plasma in modifying the nanotubes. FTIR demonstrated to be inadequate to describe the structural and chemical change of functionalized nanotubes (f-CNTs). On the contrary, Raman and XPS allowed concluding that oxygen (both during air and oxygen plasma treatment) progressively binds to the nanotubes under the form of carbonyls and carboxyls. In particular, when oxygen plasma is used, carboxylic carbons achieve the maximum concentration. Furthermore, the ratio of the intensity of the two characteristic bands of CNTs (ID/IG ratio) can be definitely used as a qualitative and comparative measure of the functionalization level of $\mathrm{f}$-CNTs.

A selection of $\mathrm{f}$-CNTs was melt blended to polyamide 6 (PA6) and the related composites were fully characterized 
from a mechanical and morphological point of view. The elastic modulus and the impact strength are about doubled if compared with the neat matrix when oxygen $\mathrm{f}$-CNTs were used. The mechanical results were corroborated by SEM observations that indicate the presence of bundles and generally a bad dispersion and adhesion on unmodified CNTs and, conversely, a good dispersion and wetting when using f-CNTs.

Received: July 11, 2011; Revised: December 19, 2011; Accepted: January 5, 2012; DOI: 10.1002/ppap.201100140

Keywords: carbon nanotubes; nanoparticles; plasma etching; polymers; XPS

[1] S. Iijima, Nature 1991, 354, 56.

[2] N. G. Sahoo, S. Rana, J. Whan Cho, L. Li, S. H. Chan, Progr. Polym. Sci. 2010, 35, 837.

[3] D. B. Mawhinney, V. Naumenko, A. Kuznetsova, J. T. Yates, J. Liu, R. E. Smalley, Chem. Phys. Lett. 2000, 324, 213.

[4] S. Niyogi, M. A. Hamon, H. Hu, B. Zhao, P. Bhowmik, R. Sen, M. E. Itkis, R. C. Haddon, Acc Chem. Res. 2002, 35, 1105.

[5] H. Park, J. Zhao, J. P. Lu, Nano Lett. 2006, 6, 916.

[6] J. F. Friedrich, S. Wettmarshausen, S. Hanelt, R. Mach, R. Mix, E. B. Zeynalov, A. Meyer-Plath, Carbon 2010, 48, 3884.

[7] J. Liu, A. G. Rinzler, H. Dai, J. H. Hafner, R. K. Bradley, P. J. Boul, Science 1998, 280, 1253.

[8] V. Georgakilas, K. Kordatos, M. Prato, D. M. Guldi, M. Holzingger, A. Hirsch, J. Am Chem. Soc. 2002, 124, 760.

[9] O. Meincke, D. Kaempfer, H. Weickmann, C. Friedrich, M. Vathauer, H. Warth, Polymer 2004, 45, 739.
[10] J. Zhang, H. Zou, O. Oing, Y. Yang, O. Li, Z. Liu, X. Guo, Z. Du, J. Phys. Chem. B 2003, 107, 3712.

[11] T. Liu, I. Y. Phang, L. Shen, S. Y. Chow, W. Zhang, Macromolecules 2004, 37, 7214.

[12] G. X. Chen, H. S. Kim, B. H. Park, J. S. Yoon, Polymer 2006, 47, 4760.

[13] H. Xia, O. Wang, G. Qiu, Chem. Mater. 2003, 15, 3879.

[14] D. A. Shirley, Phys. Rev. B 1972, 5, 4709.

[15] J. F. Moulder, W. F. Stickle, P. E. Sobol, K. D. Bomben, in Handbook of X-ray Photoelectron Spectroscopy, J. Chastain, Ed., Physical Electronics, Eden Prairie, MN 1992.

[16] D. Briggs, J. C. Riviere, in Practical Surface Analysis, D. Briggs, M. P. Seah, Eds., Wiley, New York 1983.

[17] L. Jiang, L. Gao, J. Sun, J. Colloid Interf. Sci. 2003, 260, 89.

[18] C. Chen, B. Liang, A. Ogino, X. Wang, M. Nagatsu, J. Phys. Chem. C 2009, 113, 7659.

[19] K. Hata, D. N. Futaba, K. Mizuno, T. Namai, M. Yumura, S. Iijima, Science 2004, 306, 1362.

[20] J. A. Kim, D. G. Seong, T. J. Kang, J. R. Youn, Carbon 2006, 44, 1898.

[21] C. C. Li, J. L. Lin, S. J. Huang, J. T. Lee, C. H. Chen, Colloids Surf. A Physicochem. Eng. Aspects 2007, 297, 275.

[22] J. H. Lehman, M. Terrones, E. Mansfield, K. E. Hurst, V. Meunier, Carbon 2011, 49, 2581.

[23] G. Socrates, Infrared and Raman Characteristic Group Frequencies, John Wiley \& Sons Ltd, Chichester (UK) 2001.

[24] M. S. Dresselhaus, A. Jorio, M. Hofmann, G. Dresselhaus, R. Saito, Nano Lett. 2010, 10, 751.

[25] J. Y. Yook, J. Jun, S. Kwak, Appl. Surf. Sci. 2010, 256, 6941.

[26] S. Wang, T. Wang, Y. Li, X. Zhao, J. Han, J. Wang, Plasma Sci. Technol. 2007, 9, 194.

[27] C. Fernandez-Sanchez, E. Pellicer, J. Orozco, C. JimenezJorquera, L. M. Lechuga, E. Mendoza, Nanotechnology 2009, 20, 335501.

[28] A. Maiti, J. Wescott, P. Kung, Mol. Simul. 2005, 31, 143.

[29] M. R. Nyden, S. I. Stoliarov, Polymer 2008, 49, 635.

[30] M. Morcom, K. Atkinson, G. P. Simon, Polymer 2010, 51, 3540. 